\title{
Volume stability of the augmented sinus using a collagenated bovine bone mineral grafted in case of a perforated Schneiderian membrane: An experimental study in rabbits
}

\author{
Paik, Jeong-Won ; Cha, Jae-Kook ; Paeng, Kyeong-Won ; Kim, Myong-Ji ; Thoma, Daniel S ; Jung, Ronald E ; \\ Jung, Ui-Won
}

\begin{abstract}
Objectives: To determine the volume stability of a sinus augmented with a collagenated bovine bone mineral (CBBM) in case of an intact or perforated Schneiderian membrane (SM). Materials and methods: A bilateral sinus augmentation procedure was performed in eight rabbits. The SM was intentionally perforated in one side (SMP group), while it remained intact in contra-lateral side (control group) and the same amount of CBBM was then grafted. At 12 weeks, the animals were euthanized for radiographic and histomorphometric analyses. Results: The augmented volume did not differ significantly between the two groups: $262.2 \pm 32.1 \mathrm{~mm} 3$ in SMP group and $261.9 \pm 48.5 \mathrm{~mm} 3$ in the control group $(\mathrm{p}=.959)$. There was no significant difference in the total augmented area: $24.7 \pm 5.2 \mathrm{~mm} 2$ in SMP group and $23.2 \pm 2.9 \mathrm{~mm} 2$ in the control group $(\mathrm{p}=.773)$. The areas of newly formed bone also did not differ significantly between the two groups, but was significantly lower at the centre of the augmented region than in the region of the surgical window in both groups $(\mathrm{p}<.05)$. Conclusion: A perforation of the SM in a rabbit model does neither impact the augmented volume nor new bone formation following grafting of the sinus with a CBBM. Keywords: Schneiderian membrane; animal study; volume stability; xenograft. (C) 2020 John Wiley Sons A/S. Published by John Wiley Sons Ltd.
\end{abstract}

DOI: https://doi.org/10.1111/jcpe.13273

Posted at the Zurich Open Repository and Archive, University of Zurich

ZORA URL: https://doi.org/10.5167/uzh-187816

Journal Article

Accepted Version

Originally published at:

Paik, Jeong-Won; Cha, Jae-Kook; Paeng, Kyeong-Won; Kim, Myong-Ji; Thoma, Daniel S; Jung, Ronald E; Jung, Ui-Won (2020). Volume stability of the augmented sinus using a collagenated bovine bone mineral grafted in case of a perforated Schneiderian membrane: An experimental study in rabbits. Journal of Clinical Periodontology, 47(5):649-656.

DOI: https://doi.org/10.1111/jcpe.13273 
DR. DANIEL STEFAN THOMA (Orcid ID : 0000-0002-1764-7447)

PROF. UI-WON JUNG (Orcid ID : 0000-0001-6371-4172)

Article type $\quad$ : Original Article Pre-Clinical Sciences

Volume stability of the augmented sinus using a collagenated bovine bone mineral grafted in case of a perforated Schneiderian membrane: An experimental study in rabbits

Jeong-Won Paik ${ }^{1}$, Jae-Kook Cha ${ }^{1}$, Kyeong-won Paeng ${ }^{1}$, Myong-Ji Kim ${ }^{1}$, Daniel S. Thoma ${ }^{2}$,

Ronald E. Jung ${ }^{2}$, Ui-Won Jung ${ }^{1}$

${ }^{1}$ Department of Periodontology, Research Institute for Periodontal Regeneration, Yonsei

University College of Dentistry, Seoul, Korea

${ }^{2}$ Clinic of Reconstructive Dentistry, Center of Dental Medicine, University of Zurich, Switzerland

Running title: Sinus augmentation with perforation

Keywords: xenograft; Schneiderian membrane; volume stability; animal study

\section{Correspondence address:}

Ui-Won Jung, DDS, PhD

Department of Periodontology, Yonsei University College of Dentistry

50-1 Yonsei-ro, Seodaemun-gu, Seoul 03722, Republic of Korea.

This article has been accepted for publication and undergone full peer review but has not been through the copyediting, typesetting, pagination and proofreading process, which may lead to differences between this version and the Version of Record. Please cite this article as doi: $\underline{10.1111 / \text { jepe. } 13273}$

This article is protected by copyright. All rights reserved 
Tel.: +82-2-22283185

Fax: +82-2-3920398

E-mail: drjew@yuhs.ac

\section{Conflicts of interest}

The authors declare that they have no conflicts of interest.

\section{Sources of funding}

This work was supported by the National Research Foundation of Korea (NRF) grant funded by the Korea government (Ministry of Science, ICT \& Future Planning) (No. NRF2017R1A2B2002537). 


\section{Abstract}

Objectives: To determine the volume stability of a sinus augmented with a collagenated bovine bone mineral (CBBM) in case of an intact or perforated Schneiderian membrane (SM).

Materials and Methods: A bilateral sinus augmentation procedure was performed in eight rabbits. The SM was intentionally perforated in one side (SMP group), while it remained intact in contralateral side (control group) and the same amount of CBBM was then grafted. At 12 weeks, the animals were euthanized for radiographic and histomorphometric analyses.

Results: The augmented volume did not differ significantly between the two groups: $262.2 \pm 32.1 \mathrm{~mm}^{3}$ in SMP group and $261.9 \pm 48.5 \mathrm{~mm}^{3}$ in the control group ( $p=0.959$ ). There was no significant difference in the total augmented area: $24.7 \pm 5.2 \mathrm{~mm}^{2}$ in SMP group and $23.2 \pm 2.9 \mathrm{~mm}^{2}$ in the control group ( $p=0.773$ ). The areas of newly formed bone also did not differ significantly between the two groups, but was significantly lower at the center of the augmented region than in the region of the surgical window in both groups $(p<0.05)$.

Conclusion: A perforation of the SM in a rabbit model, does neither impact the augmented volume nor new bone formation following grafting of the sinus with a CBBM.

\section{Introduction}

Sinus augmentation is a highly predictable surgical technique for increasing the height of the alveolar bone in the posterior maxilla. The lateral approach for sinus augmentation has been preferred when the amount of residual bone is not sufficient to achieve primary stability of implants (Hallman, Cederlund et al. 2001, Hallman, Sennerby et al. 2002, Pjetursson, Tan et al. 2008, Tan, Lang et al. 2008, Tukel and Tatli 2018).

While sinus augmentation is considered a predictable procedure with high implant survival rates, intraoperative or post-operative complications can still occur (Danesh-Sani, Loomer et al. 2016, Stacchi, Andolsek et al. 2017). It has been reported that the most-frequent intraoperative complication is perforation of the Schneiderian membrane (SM), with a reported incidence rate ranging from 10\% to 55\% (Schwarz, Schiebel et al. 2015, Al-Dajani 2016). The SM can be perforated due to anatomic reasons, surgical risk factors or pathophysiologic factors (Favero, Lang 
et al. 2016). There are several treatment options for a perforated SM, including tissue fibrin glue, suturing, human lamellar bone sheets with or without a buccal fat-pad flap, and covering with a resorbable barrier membrane (Vlassis and Fugazzotto 1999, Hernandez-Alfaro, Torradeflot et al. 2008). Among these treatment modalities, resorbable collagen membrane has been frequently used for small to intermediate-sized SM perforations to prevent the loss of the graft materials (Fugazzotto and Vlassis 2003, Barone, Santini et al. 2006).

The primary purpose of the collagen membrane is to contain the particulate graft material so that it does not become scattered through the perforation as well as to seal the perforated area to prevent the invasion of sinus bacteria (Nolan, Freeman et al. 2014). However, the effects of a collagen membrane on the subsequent healing are controversially discussed (Testori, Wallace et al. 2008, Froum, Khouly et al. 2013). The natural healing process of a perforated SM begins with the migration of new cells from the undamaged area, but little is known about how this is affected by the presence of a collagen membrane. It is also unclear how a collagen membrane affects the osteogenic potential of the SM. In addition, using a collagen membrane increases the surgical time and cost.

The use of block-type graft material could be an alternative to particulate bone with the collagen membrane for a sinus with a perforated SM. Due to its size, the block bone could remain in the grafted area and not pass through the perforation even when a collagen membrane is not applied (Pikos 1999, Chaushu, Mardinger et al. 2009).

Collagenated bovine bone mineral (CBBM), which is an altered formulation of deproteinized bovine bone mineral (DBBM), is available in a block form that is stabilized in $10 \%$ porcine type-I collagen matrix. DBBM is well defined as a slower resorbing and highly osteoconductive material, which makes it one of the best-documented bone substitutes for use in maxillary sinus augmentation. Although excellent histologic results have been reported when using CBBM in the extraction socket of beagle dogs, few studies have investigated the use of CBBM in the maxillary sinus (Araujo, Liljenberg et al. 2010). Chiapasco et al. also suggested that CBBM could be advantageous in maxillary sinus augmentation when the common surgical complication of SM perforation occurs, but there are also no reports on the bone healing of the perforated SM using this material (Chiapasco, Casentini et al. 2009). 
Hence, the purpose of the present study was to determine the volume stability of a sinus augmented with a collagenated bovine bone mineral (CBBM) in case of an intact or perforated Schneiderian membrane (SM). The null hypothesis tested was that perforation of the SM during sinus augmentation did not affect the volume stability of grafted CBBM in a rabbit sinus model.

\section{Materials and Methods}

This study was designed following the modified ARRIVE guidelines for preclinical research (Vignoletti and Abrahamsson 2012).

\section{Experimental animals, housing and husbandary}

Eight male adult New Zealand White rabbits weighing 2.5-3.0 kg were selected for these experiments. The animals were housed individually following standard laboratory conditions, fed a standard laboratory pellet diet and water ad libitum. The selection procedure, care, surgical protocols and preparation procedures were applied to the animals in accordance with the guidelines of the Institutional Animal Care and Use Committee of Yonsei Medical Center, Seoul, Korea (Approval No. 2018-0070).

\section{Sample size}

Sample size calculation was based on estimations from a previous preclinical study(Yon, Lee et al, 2015 ) using the changes in a histometrical difference in new bone area of $10 \%$ with $95 \%$ power, an alpha level of 0.05 , and a within-group standard deviation of 5\%. A sample size of 8 animals was calculated per experimental group.

\section{Study design}

Sinus augmentation procedures were performed on both sides of the maxilla in each rabbit according to a split-mouth design. Following elevation of the SM, each sinus was assigned to one of the following groups: (i) SM perforation followed by grafting half of a 250-mg CBBM block (SMP group) and (ii) grafting the same amount of the same material but without perforating the SM (control group). The group allocation (left/right sinus) was determined by a random number produced by computer software(SPSS 15.0, SPSS, Chicago, IL, USA). Treatments allocation was unveiled only following completion of the site preparation. 


\section{Surgical procedure}

The surgical procedures were performed under general anaesthesia induced by an intramuscular injection of a mixture of ketamine hydrochloride (Ketalar, Yuhan, Seoul, Korea) and xylazine (Rompun, Bayer Korea, Seoul, Korea). The surgical site was shaved and then disinfected using iodine solution. Local anaesthesia using $2 \%$ lidocaine (lidocaine $\mathrm{HCl}$, Huons, Seoul, Korea) was applied at the surgical site. A perpendicular incision was made along the sagittal line of the nasal bone and the full thickness of skin including the periosteum was separated from the bone. Windows were made in the lateral walls of both sinuses using a 5.5-mm-diameter circular reamer (C-reamer, NeoBiotech, Seoul, Korea) and the thin bone disc was carefully removed. In SMP group, the SM was intentionally perforated using a 3-mm-diameter drill after it had been carefully elevated, subsequently, the experimental material (125 mg of CBBM; Bio-Oss Collagen ${ }^{\circledR}$, Geistlich Pharma, Wolhusen, Switzerland) was placed in the sinus. In the control group, the same amount of CBBM was placed without perforating the SM (Figure 1). After grafting, the removed bone disc was repositioned to cover the antrostomies without collagen membrane and primary wound closure was achieved using absorbable monofilament (6-0 Monosyn ${ }^{\circledR}$, B-Braun, Aesculap, Center Valley, PA, USA). Skin sutures were removed after 7 days. The animals were carefully monitored during the assigned healing period, and antibiotics (enrofloxacin; $5 \mathrm{mg} / \mathrm{kg}$ Baytril, Bayer Korea, Seoul, Korea) and analgesics $(0.5 \mathrm{mg} / \mathrm{kg}$ ketorolac; Keromin, Hana Pharm, Seoul, Korea) were administered once daily for 5 days. The animals were euthanized at 12 weeks postoperatively.

\section{Micro-computed tomographic analysis}

Block sections of the sinus sites were dissected along with adjacent tissue and fixed in $10 \%$ neutral buffered formalin for 10 days. The fixed specimens were then scanned using high-energy spiral-scan micro-computed tomography (micro-CT; SkyScan 1173, Bruker Micro-CT, Kontich, Belgium) at a resolution of $14.91 \mu \mathrm{m}$ (achieved using $130 \mathrm{kV}$ and $60 \mu \mathrm{A}$ ). The acquired data were processed and reconstructed into 3D images using NRecon software (Bruker Micro-CT). The sinuses were identified by applying a threshold to the greyscale values. Radiographic measurements were made twice by a blinded, experienced examiner using an automated digital method. The total augmented volume (TAV) was determined, and then the new bone volume 
(NBV), residual bone graft material volume (RMV) and the non-mineralized tissue volume (NMV) were measured within the TAV. All of the volumes were measured in cubic millimetres.

\section{Histomorphometric analysis}

Micrographs were taken at a magnification of $\times 12.5$ and combined to enable visualization of the entire sinus. Histomorphometric measurements were conducted by a blinded, experienced examiner using a personal-computer-based image-analysis system (Photoshop, Adobe, San Jose, CA, USA).

The following measurements were recorded:

Total augmented area (TAA): the area surrounded by the antral bone wall, the SM and the surgical access window.

New bone area (NBA): the area and the percentage of newly formed bone within the TAA.

Residual graft material area (RMA): the area and percentage of CBBM within the TAA.

Non-mineralized tissue area (NMA): the area and percentage of non-mineralized tissue within the TAA.

TAA was measured in square millimetres, while NBA and RMA were measured in both square millimetres and as percentages.

In order to evaluate the homogeneity of regenerated bone in the grafted sinus area, the following three regions of interest (ROIs) (size: $1.0 \mathrm{~mm} \times 1.0 \mathrm{~mm}$ ) were defined in accordance with previous studies (Kim, Cha et al. 2015, Yon, Lee et al. 2015): close to the surgical access window (ROI_W), at the center of the augmented sinus (ROI_C) and close to the SM (ROI_M). The absolute values and the relative percentages of NBA and RMA were calculated within each ROI.

\section{Experimental outcomes}

The primary outcomes of this study were TAV and NBA, while the other histomorphometric parameters were measured as the secondary outcomes. 


\section{Statistical analyses}

Statistical analyses were performed using SPSS software (version 21.0, SPSS, Chicago, IL, USA).

Data are presented as mean $\pm \mathrm{SD}$ and median values. The Mann-Whitney $U$ test was used to compare SMP and control groups, and the Kruskal-Wallis test was used for intragroup comparisons. Post hoc comparisons were made using the Bonferroni correction. The criterion for statistical significance was set at $p<0.05$.

\section{Results}

\section{Clinical observations}

All animals were healthy and wound healing was uneventful throughout the entire experimental period, with no adverse reactions such as pus discharge, hematoma or swelling.

After sacrificing each animal, both maxillary sinuses appeared to be covered with soft tissues, and no animals had any unhealed perforation sites. It was difficult to distinguish between the two groups visually (Figure 1).

\section{Micro-computed tomographic analysis}


Each augmented sinus had a dome-shape appearance in the 3D reconstructed views(Figure 2). The grafted material appeared to have maintained its original volume without scattering in both groups. The augmented forms were very similar in SMP and control groups, and it was not easy to distinguish between the two groups radiographically.

In a cross-sectional view, the graft material adhered closely to the native sinus bone wall and the radiopaque newly formed bone was observed between graft particles. The new bone appeared to have formed mainly around the native sinus bone wall and the SM, with less in the center of the sinus. This pattern was similar in both groups.

TAV did not differ significantly between SMP and control groupsNBV, NMV and RMV also did not differ significantly between SMP and control groups $(p>0.05)$ (Table 1).

\section{Histologic findings and histomorphometric analysis}

Similar to the radiographic findings, the maxillary sinus cavity appeared augmented with a dome shape in the histomorphometric analysis, and the surgical access window was completely closed by new bone in all specimens. Most of the newly formed bone was observed adjacent to the native bone near the access window, with some new bone formation adjacent to the SM. There was less new bone formation in the center of the augmented sinus, with this region mainly being filled with residual graft materials and fibrovascular tissues in both groups(Figure 3).

The continuity of the SM had recovered in SMP group, but some of the grafted material was embedded within the SM. The epithelium of the perforated SM was properly repaired by a dense connective tissue, which was lined with atrophied cilia. A few goblet cells were also found in the dense connective tissues in SMP group, but serous glands, which were evident in the control group, were not found at the perforated site (Figure 4a). On the other hand, the intact SM in the control group was lined with respiratory epithelium composed of ciliated, goblet and basal cells (Figure $4 b)$.

The healing outcomes are presented at higher magnifications in Figure 4. TAA did not differ significantly between SMP $\left(24.7 \pm 5.2 \mathrm{~mm}^{2}\right)$ and control $\left(23.2 \pm 2.9 \mathrm{~mm}^{2}\right)$ groups. NBA and RBA also did not differ significantly between the two groups ( $p>0.05$; Table 2$)$. 
Intragroup regional comparisons revealed that there was significantly greater new bone formation in ROI_W adjacent to the native sinus wall than in ROI_C in both groups $(p<0.05)$. The NBAs in SMP and control groups were $34.6 \%$ and $26.2 \%$, respectively, in ROI_W, and $20.5 \%$ and $18.8 \%$ in ROI_C. There appeared to be slightly more new bone formation in ROI_M than in ROI_C, but the difference was not statistically significant ( $p=0.063$; Table 2, Figure 5).

\section{Discussion}

This study evaluated the volume stability of the augmented sinus using a CBBM block under two conditions: intact or perforated SM. The null hypothesis tested was that perforation of the SM during sinus augmentation does not affect the volume stability of grafted CBBM in a rabbit sinus model. The results demonstrated that SMP group showed similar quantity and quality of augmented bone compared to the control group, and so the null hypothesis was accepted.

While maxillary sinus augmentation is known to be a predictable procedure, the optimal criteria for success have not been established, yet. Most previous clinical studies have used assessments of the survival rate of implants when evaluating the success of sinus augmentation, while other studies have analyzed the volume stability or performed histomorphometric measurements (ChoLee, Naval-Gias et al. 2010, Mazzocco, Lops et al. 2014, Sbordone, Toti et al. 2014, Danesh-Sani, Engebretson et al. 2017, de Almeida Ferreira, Martinelli et al. 2017).

Volume stability should be achieved after maxillary sinus augmentation in order to allow the placement of implants with adequate diameters and lengths. Moreover, different bone graft materials have been used to maintain the augmented volume stability (Cha, Kim et al. 2019). The present study chose CBBM as the graft material with the aim of preventing any scattering of grafted bone through the perforated SM. CBBM shows excellent results for extraction socket management, but little is known about the healing outcomes in maxillary sinus augmentation. The volume stability of graft material in extraction sockets is not a major concern because these materials are surrounded by hard tissues such as alveolar bone. However, the results obtained for the volume maintenance when using CBBM varied with the pathophysiologic characteristics at the defect site. In a previous study in a dog model, CBBM could not maintain the volume when it was used as an onlay graft material (Cha, Kim et al. 2019). The maxillary sinus is a unique space in which graft materials are placed between hard and soft tissues and the presence of constant respiratory air pressure makes it difficult to maintain volume stability. CBBM is a soft type of 
block bone consisting of DBBM and the stabilizing type I collagen, which is known to degrade within 1-2 weeks, and this will result in the TAV deteriorating during the healing period. In the present study, the amount of CBBM used $(125 \mathrm{mg})$ corresponded to a typical volume of 250 300 $\mathrm{mm}^{3}$, which resulted in TAV being more than $260 \mathrm{~mm}^{3}$ in both groups. These findings indicate that while the collagen portion is rapidly resorbed, CBBM maintains the volume stability even in the presence of the respiratory air pressure.

There are two major concerns for clinicians about a perforated SM. The first is the bone quantity. The augmented material can be scattered throughout the perforated area, which may introduce foreign-body reactions in the maxillary sinus cavity as well as decrease the augmented bone volume. Micro-CT analysis provides highly meaningful data for assessing volume stability, and TAV is an important parameter that can only be measured in animal studies.

TAV did not differ significantly between the present test and control groups, at $262.2 \pm 32.1 \mathrm{~mm}^{3}$ and $261.9 \pm 48.5 \mathrm{~mm}^{3}$, respectively. This suggests that CBBM remained in its block form and so did not scatter to the perforated site. It is assumed that the epithelial lining formed before the resorption of the collagen portion, which acts as a binder that maintains its block form. (Forsgren, Stierna et al. 1993) reported that poorly differentiated epithelial linings could be found as early as 1 week after the SM was perforated in a rabbit. Further differentiation toward a ciliated epithelium occurred after 2 weeks, and fibrosis, which is the most-prominent finding in the injured SM, progressed but the serous glands in the lamina propria were replaced by scar tissue. Those previous results are in agreement with the present finding of the SM being healed by dense connective tissue. These results also suggest that CBBM can be used as an alternative in SMperforated maxillary sinus augmentation without requiring an additional collagen membrane.

The second concern is about the bone quality, because a compromised bone quality next to the perforated area may impair osseointegration. In the present study, a radiographic cross-sectional view revealed no significant differences in NBV, RMV or NMV. The histomorphometric analysis also showed that NBA and RMA did not differ significantly between SMP and control groups. These results suggest that the presence of a small perforation in the SM with a diameter of around $3 \mathrm{~mm}$ did not significantly affect the new bone formation when using CBBM without a collagen membrane. 
Applying a resorbable collagen membrane to a perforated SM has been demonstrated to achieve a physical sealing effect, but its effects on healing of the SM and new bone formation are still unclear. A previous study found delayed new bone formation when using a collagen membrane and particulate bone on a perforated SM in a rabbit sinus model (Lim, Son et al. 2018). It is assumed that the collagen membrane may impair the natural healing process of the SM by delaying the migration of undifferentiated basal cells from the adjacent untraumatized area or obstructing the osteogenic potential of the SM.

The present study divided the augmented area into three ROIs: next to the SM, at the center and at the surgical access window. NBA was significantly lower in ROC_C than in ROI_W in both groups, but it did not differ significantly between ROI_W and ROI_M. These results confirm that the SM as well as native sinus bone wall have osteogenic potential.

Previous studies suggested that osteogenic cells originate from the native sinus bone wall (Boyne and James 1980, Misch and Dietsh 1991). Although the osteogenic potential of the SM remains controversial, the results obtained in the present study support that the SM also has osteogenic potential to enhance the new bone formation of augmented bone. Kim et al. (2014) reported the osteogenic potential of the SM by revealing high ALP activity as well as mRNA expression in SM cells. Srouji et al. (2010) evaluated the osteogenic potential of the SM and reported ectopic bone formation in conjunction with an osteoconductive scaffold near the SM (Srouji, Ben-David et al. 2010, Kim, Kwon et al. 2014). The present study found that the use of CBBM resulted in a considerable amount of new bone next to the membrane in both groups. This demonstrates that using CBBM for a perforated SM does not have a negative effect on new bone formation. Within the limitations of this study, it can be concluded that a CBBM block can maintain the volume with similar bone formation in an SM with a 3-mm-diameter perforation in a rabbit sinus model.

\section{Conclusion}

A perforation of the SM in a rabbit model, does neither impact the augmented volume nor new bone formation following grafting of the sinus with a CBBM block. 


\section{References}

Al-Dajani, M. (2016). "Incidence, Risk Factors, and Complications of Schneiderian Membrane Perforation in Sinus Lift Surgery: A Meta-Analysis." Implant Dent 25(3): 409-415.

Araujo, M. G., B. Liljenberg and J. Lindhe (2010). "Dynamics of Bio-Oss Collagen incorporation in fresh extraction wounds: an experimental study in the dog." Clin Oral Implants Res 21(1): 5564.

Barone, A., S. Santini, L. Sbordone, R. Crespi and U. Covani (2006). "A clinical study of the outcomes and complications associated with maxillary sinus augmentation." Int J Oral Maxillofac Implants 21(1): 81-85.

Boyne, P. J. and R. A. James (1980). "Grafting of the maxillary sinus floor with autogenous marrow and bone." J Oral Surg 38(8): 613-616.

Cha, J. K., C. Kim, H. C. Pae, J. S. Lee, U. W. Jung and S. H. Choi (2019). "Maxillary sinus augmentation using biphasic calcium phosphate: dimensional stability results after 3-6 years." J Periodontal Implant Sci 49(1): 47-57.

Chaushu, G., O. Mardinger, S. Calderon, O. Moses and J. Nissan (2009). "The use of cancellous block allograft for sinus floor augmentation with simultaneous implant placement in the posterior atrophic maxilla." J Periodontol 80(3): 422-428.

Chiapasco, M., P. Casentini and M. Zaniboni (2009). "Bone augmentation procedures in implant dentistry." Int J Oral Maxillofac Implants 24 Suppl: 237-259.

Cho-Lee, G. Y., L. Naval-Gias, S. Castrejon-Castrejon, A. L. Capote-Moreno, R. Gonzalez-Garcia, J. Sastre-Perez and M. F. Munoz-Guerra (2010). "A 12-year retrospective analytic study of the implant survival rate in 177 consecutive maxillary sinus augmentation procedures." Int J Oral Maxillofac Implants 25(5): 1019-1027.

Danesh-Sani, S. A., S. P. Engebretson and M. N. Janal (2017). "Histomorphometric results of different grafting materials and effect of healing time on bone maturation after sinus floor augmentation: a systematic review and meta-analysis." J Periodontal Res 52(3): 301-312. 
Danesh-Sani, S. A., P. M. Loomer and S. S. Wallace (2016). "A comprehensive clinical review of maxillary sinus floor elevation: anatomy, techniques, biomaterials and complications." $\mathrm{Br} \mathrm{J}$ Oral Maxillofac Surg 54(7): 724-730.

de Almeida Ferreira, C. E., C. B. Martinelli, A. B. Novaes, Jr., T. B. Pignaton, C. C. Guignone, A. L. Goncalves de Almeida and E. Saba-Chujfi (2017). "Effect of Maxillary Sinus Membrane Perforation on Implant Survival Rate: A Retrospective Study." Int J Oral Maxillofac Implants 32(2): 401-407.

Favero, V., N. P. Lang, L. Canullo, J. Urbizo Velez, F. Bengazi and D. Botticelli (2016). "Sinus floor elevation outcomes following perforation of the Schneiderian membrane. An experimental study in sheep." Clin Oral Implants Res 27(2): 233-240.

Forsgren, K., P. Stierna, J. Kumlien and B. Carlsoo (1993). "Regeneration of maxillary sinus mucosa following surgical removal. Experimental study in rabbits." Ann Otol Rhinol Laryngol 102(6): 459-466.

Froum, S. J., I. Khouly, G. Favero and S. C. Cho (2013). "Effect of maxillary sinus membrane perforation on vital bone formation and implant survival: a retrospective study." J Periodontol 84(8): 1094-1099.

Fugazzotto, P. A. and J. Vlassis (2003). "A simplified classification and repair system for sinus membrane perforations." J Periodontol 74(10): 1534-1541.

Hallman, M., A. Cederlund, S. Lindskog, S. Lundgren and L. Sennerby (2001). "A clinical histologic study of bovine hydroxyapatite in combination with autogenous bone and fibrin glue for maxillary sinus floor augmentation. Results after 6 to 8 months of healing." Clin Oral Implants Res 12(2): 135-143.

Hallman, M., L. Sennerby and S. Lundgren (2002). "A clinical and histologic evaluation of implant integration in the posterior maxilla after sinus floor augmentation with autogenous bone, bovine hydroxyapatite, or a 20:80 mixture." Int J Oral Maxillofac Implants 17(5): 635-643. Hernandez-Alfaro, F., M. M. Torradeflot and C. Marti (2008). "Prevalence and management of Schneiderian membrane perforations during sinus-lift procedures." Clin Oral Implants Res 19(1): 91-98.

Kim, J. S., J. K. Cha, A. R. Cho, M. S. Kim, J. S. Lee, J. Y. Hong, S. H. Choi and U. W. Jung (2015). "Acceleration of Bone Regeneration by BMP-2-Loaded Collagenated Biphasic Calcium Phosphate in Rabbit Sinus." Clin Implant Dent Relat Res 17(6): 1103-1113. 
Kim, M. S., J. Y. Kwon, J. S. Lee, J. S. Song, S. H. Choi and U. W. Jung (2014). "Low-dose recombinant human bone morphogenetic protein-2 to enhance the osteogenic potential of the Schneiderian membrane in the early healing phase: in vitro and in vivo studies." J Oral Maxillofac Surg 72(8): 1480-1494.

Lim, H. C., Y. Son, J. Y. Hong, S. I. Shin, U. W. Jung and J. H. Chung (2018). "Sinus floor elevation in sites with a perforated schneiderian membrane: What is the effect of placing a collagen membrane in a rabbit model?" Clin Oral Implants Res 29(12): 1202-1211.

Mazzocco, F., D. Lops, L. Gobbato, A. Lolato, E. Romeo and M. del Fabbro (2014). "Threedimensional volume change of grafted bone in the maxillary sinus." Int J Oral Maxillofac Implants 29(1): 178-184.

Misch, C. E. and F. Dietsh (1991). "Autogenous bone grafts for endosteal implants--indications and failures." Int J Oral Implantol 8(1): 13-20.

Nolan, P. J., K. Freeman and R. A. Kraut (2014). "Correlation between Schneiderian membrane perforation and sinus lift graft outcome: a retrospective evaluation of 359 augmented sinus." J Oral Maxillofac Surg 72(1): 47-52.

Pikos, M. A. (1999). "Maxillary sinus membrane repair: report of a technique for large perforations." Implant Dent 8(1): 29-34.

Pjetursson, B. E., W. C. Tan, M. Zwahlen and N. P. Lang (2008). "A systematic review of the success of sinus floor elevation and survival of implants inserted in combination with sinus floor elevation." J Clin Periodontol 35(8 Suppl): 216-240.

Sbordone, C., P. Toti, F. Guidetti, L. Califano, G. Pannone and L. Sbordone (2014). "Volumetric changes after sinus augmentation using blocks of autogenous iliac bone or freeze-dried allogeneic bone. A non-randomized study." J Craniomaxillofac Surg 42(2): 113-118.

Schwarz, L., V. Schiebel, M. Hof, C. Ulm, G. Watzek and B. Pommer (2015). "Risk Factors of Membrane Perforation and Postoperative Complications in Sinus Floor Elevation Surgery: Review of 407 Augmentation Procedures." J Oral Maxillofac Surg 73(7): 1275-1282.

Srouji, S., D. Ben-David, R. Lotan, M. Riminucci, E. Livne and P. Bianco (2010). "The innate osteogenic potential of the maxillary sinus (Schneiderian) membrane: an ectopic tissue transplant model simulating sinus lifting." Int J Oral Maxillofac Surg 39(8): 793-801.

Stacchi, C., F. Andolsek, F. Berton, G. Perinetti, C. O. Navarra and R. Di Lenarda (2017).

"Intraoperative Complications During Sinus Floor Elevation with Lateral Approach: A Systematic Review." Int J Oral Maxillofac Implants 32(3): e107-e118. 
Tan, W. C., N. P. Lang, M. Zwahlen and B. E. Pjetursson (2008). "A systematic review of the success of sinus floor elevation and survival of implants inserted in combination with sinus floor elevation. Part II: transalveolar technique." J Clin Periodontol 35(8 Suppl): 241-254.

Testori, T., S. S. Wallace, M. Del Fabbro, S. Taschieri, P. Trisi, M. Capelli and R. L. Weinstein (2008). "Repair of large sinus membrane perforations using stabilized collagen barrier membranes: surgical techniques with histologic and radiographic evidence of success." Int J Periodontics Restorative Dent 28(1): 9-17.

Tukel, H. C. and U. Tatli (2018). "Risk factors and clinical outcomes of sinus membrane perforation during lateral window sinus lifting: analysis of 120 patients." Int J Oral Maxillofac Surg.

Vignoletti, F. and I. Abrahamsson (2012). "Quality of reporting of experimental research in implant dentistry. Critical aspects in design, outcome assessment and model validation." J Clin Periodontol 39 Suppl 12: 6-27.

Vlassis, J. M. and P. A. Fugazzotto (1999). "A classification system for sinus membrane perforations during augmentation procedures with options for repair." J Periodontol 70(6): 692699.

Yon, J., J. S. Lee, H. C. Lim, M. S. Kim, J. Y. Hong, S. H. Choi and U. W. Jung (2015). "Preclinical evaluation of the osteogenic potential of bone morphogenetic protein-2 loaded onto a particulate porcine bone biomaterial." J Clin Periodontol 42(1): 81-88.

\section{Figure Legends}

Fig. 1. Clinical photographs of surgical procedures.

(a) A full-thickness flap was elevated and circular bony windows with a diameter of $5.5 \mathrm{~mm}$ were made, with a 3-mm-diameter perforation also made in the Schneiderian membrane (SM) 
on one side only. (b) Placement of $125 \mathrm{mg}$ of collagenated bovine bone mineral (CBBM) on both sides. (c) After grafting, windows were repositioned to cover the graft materials.

Fig. 2. Clinical view of augmented sinus and Micro-computed tomographic images

(a) Clinical view after 12 weeks in SMP group(left arrow) and the control group(right arrow). Both maxillary sinuses are well covered with soft tissues. It was difficult to distinguish the two groups visually. (b) color-coded 3D reconstructed micro-computed tomographic images: SMP(red) and control group(green). (c) cross sectional view of the augmented sinuses.

Fig. 3. Histologic photomicrographs in SMP group(a-d) and the control group(e-h).

(a) Total area of sinus augmentation in SMP group, (b) the area close to the surgical access window (ROI_W), (c) the area at the center of the augmented sinus (ROI_C), and (d) the area close to the SM (ROI_M).

Newly formed bone (NB) was observed in the three ROI regions and mainly found adjacent to the native bone in ROI_W (b) and ROI_M (d), with little bone formation in ROI_C (c). The SM was repaired by the respiratory epithelium (RE), which consists of a dense connective tissue lined with the atrophied cilia (Masson's trichrome). (e) Total area of sinus augmentation in the control group, (f) the area in ROI_W, (g) the area in ROI_C, and (h) the area in ROI_M. NB was observed in the three ROI regions and mainly found adjacent to the native bone in ROI_W (f) and ROI_M (h), with little bone formation in ROI_C (g). Serous glands (SG) were observed in the lamina propria adjacent to the RE of the SM (Masson's trichrome).

Fig. 4. Higher magnification views of the SM.

(a) SMP group showing the repaired epithelium, with remnant grafted materials embedded within the repaired SM and dense connective tissues. The repaired SM shows no cilia and flattened goblet cells (arrow).

(b) Control group shows the intact SM and the goblet cells(white arrow) in the ciliated repiratory epithielium.Serous glands(SG) (black arrow)were observed in the lamina propria.

Fig. 5. Composition of the three ROIs in the histologic analyses.

There were no significant differences between SMP and control groups in ROI_W, ROI_M, and ROI_C $(p=0.923, p=0.564$, and $p=0.386$, respectively $)$.

* Significantly different from ROI_W in SMP group and ** significantly different from ROI_W in the control group $(p<0.05)$. 
Tables

Table 1. Composition of the total augmented volume(mean \pm SD).

\begin{tabular}{lclclc}
\hline & \multicolumn{2}{c}{ SMP } & \multicolumn{2}{c}{ Control } & $p$ value \\
\hline TAV $\left(\mathrm{mm}^{3}\right)$ & $262.19 \pm 32.08$ & $(100 \%)$ & $261.97 \pm 48.54$ & $(100 \%)$ & 0.959 \\
$\operatorname{NBV}\left(\mathrm{mm}^{3}\right)$ & $65.10 \pm 12.13$ & $(24.8 \%)$ & $66.44 \pm 11.40$ & $(25.4 \%)$ & 1.000 \\
$\operatorname{RMV}\left(\mathrm{mm}^{3}\right)$ & $66.94 \pm 17.28$ & $(23.2 \%)$ & $60.67 \pm 15.91$ & $(23.2 \%)$ & 0.574 \\
$\operatorname{NMV}\left(\mathrm{mm}^{3}\right)$ & $130.15 \pm 35.63$ & $(49.6 \%)$ & $134.86 \pm 32.20$ & $(51.5 \%)$ & 0.798
\end{tabular}

No significant difference in TAV between SMP and Control group $(p=0.959)$

TAV, total augmented volume; NBV, new bone volume; RMV, residual graft materials volume; NMV, non-mineralized tissue volume.

Table 2. Composition of the total augmented area (mean \pm SD).

\begin{tabular}{|c|c|c|c|c|c|}
\hline \multirow[b]{2}{*}{$\operatorname{TAA}\left(\mathrm{mm}^{2}\right)$} & \multirow[b]{2}{*}{ Mean $\pm \operatorname{SD}($ median $)$} & \multicolumn{2}{|c|}{ SMP } & \multicolumn{2}{|c|}{ Control } \\
\hline & & $23.2 \pm 2.9$ & $(24.1)$ & $24.7 \pm 5.2$ & $(22.2)$ \\
\hline & $\operatorname{NBA}(\%)$ & $23.4 \pm 1.1$ & $(24.8)$ & $22.0 \pm 1.1$ & $(20.3)$ \\
\hline & $\operatorname{RMA}(\%)$ & $28.0 \pm 1.3$ & $(28.7)$ & $28.3 \pm 2.3$ & $(23.6)$ \\
\hline & $\operatorname{NMA}(\%)$ & $48.6 \pm 3.9$ & $(47.2)$ & $49.7 \pm 3.4$ & $(48.7)$ \\
\hline \multirow[t]{3}{*}{ ROI_W } & $\operatorname{NBA}(\%)$ & $34.6 \pm 7.9$ & $(35.0)$ & $26.2 \pm 9.0$ & $(25.2)$ \\
\hline & $\operatorname{RMA}(\%)$ & $24.7 \pm 10.7$ & $(23.0)$ & $28.7 \pm 9.3$ & $(28.8)$ \\
\hline & $\operatorname{NMA}(\%)$ & $40.6 \pm 1.4$ & $(40.9)$ & $45.1 \pm 1.7$ & $(50.2)$ \\
\hline \multirow[t]{3}{*}{ ROI_C } & $\operatorname{NBA}(\%)$ & $20.5 \pm 6.0^{*}$ & $(21.2)$ & $18.8 \pm 7.2^{* *}$ & $(28.5)$ \\
\hline & $\operatorname{RMA}(\%)$ & $43.9 \pm 8.2$ & $(44.8)$ & $45.1 \pm 10.2$ & $(42.5)$ \\
\hline & $\operatorname{NMA}(\%)$ & $35.6 \pm 8.9$ & $(35.8)$ & $36.1 \pm 8.5$ & $(29.0)$ \\
\hline \multirow[t]{3}{*}{ ROI_M } & $\operatorname{NBA}(\%)$ & $28.5 \pm 12.0$ & $(30.1)$ & $22.2 \pm 7.7$ & $(20.0)$ \\
\hline & $\operatorname{RMA}(\%)$ & $42.3 \pm 14.6$ & $(42.8)$ & $46.1 \pm 11.5$ & $(48.5)$ \\
\hline & $\operatorname{NMA}(\%)$ & $29.2 \pm 5.7$ & $(27.1)$ & $31.7 \pm 14.4$ & $(32.7)$ \\
\hline
\end{tabular}

No significant difference in TAA between SMP and Control group $(p=0.773)$

* significantly different from ROI_W in the SMP group and ** significantly different from ROI_W in the control group $(p<0.05)$

NBA, new bone area; RMA, residual graft materials area; NMA, non-mineralized tissue area; TAA, total augmented area

ROI_W, close region to the surgical access window; ROI_C, the center region of the augmented sinus; ROI_M, close region to the SM. 


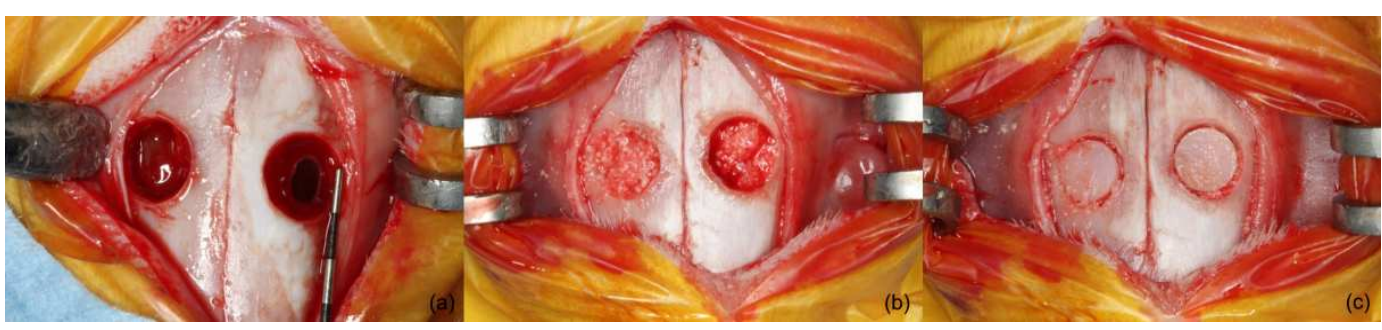

jcpe_13273_f1.tif 


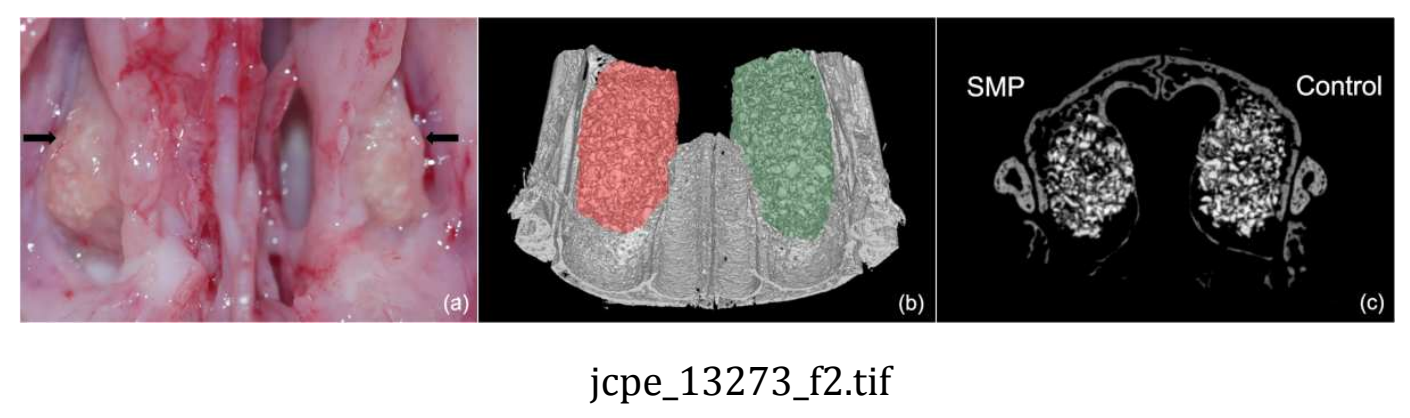

This article is protected by copyright. All rights reserved 


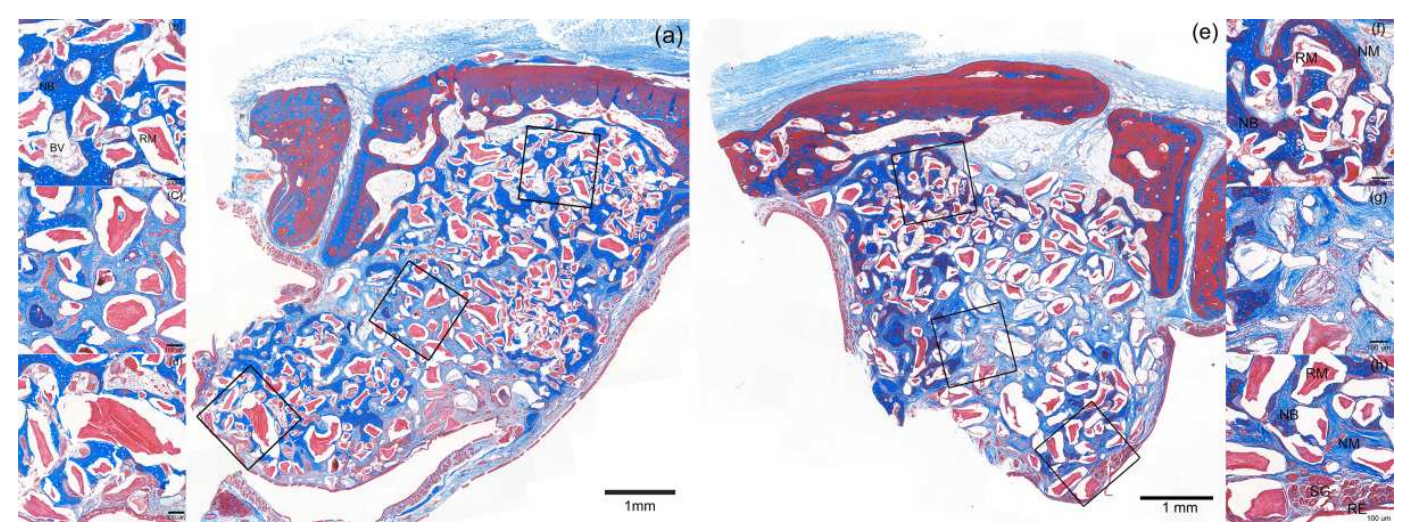

jcpe_13273_f3.tif

This article is protected by copyright. All rights reserved 


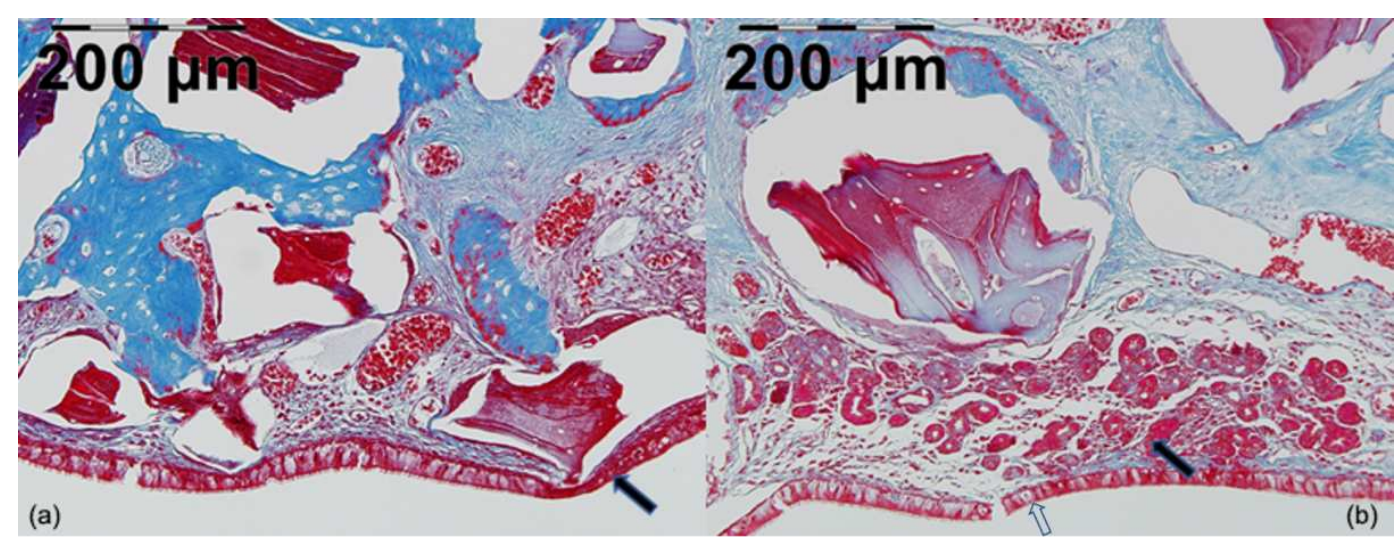

jcpe_13273_f4.tif

This article is protected by copyright. All rights reserved 

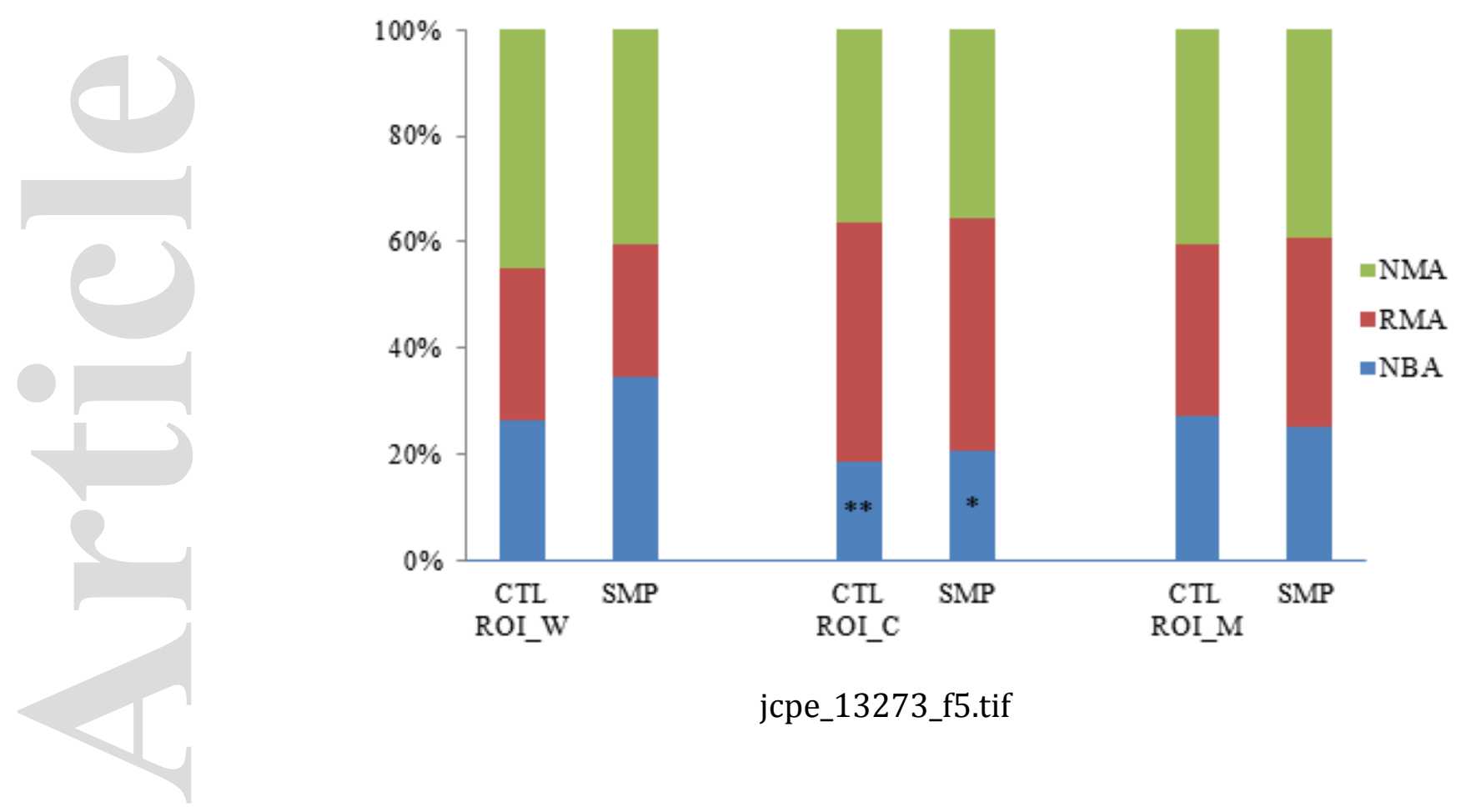

jcpe_13273_f5.tif 